\title{
ASTROMETRY WITH THE HST PLANETARY CAMERA
}

\author{
T. M. GIRARD, Y. LI AND W. F. VAN ALTENA \\ Dept. of Astronomy, Yale University \\ P.O. Box 208101, New Haven, CT 06520-8101 \\ J. M. NUNEZ \\ Univ. of Barcelona, Spain \\ G. F. BENEDICT, R. L. DUNCOMBE, P. D. HEMENWAY, \\ W. H. JEFFERYS, B. MCARTHUR, J. MCCARTNEY, E. NELAN, \\ P. SHELUS, D. STORY AND A. L. WHIPPLE \\ Univ. of Texas, USA \\ O. G. FRANZ AND L. W. WASSERMAN \\ Lowell Obs., USA \\ AND \\ L. W. FREDRICK \\ Univ. of Virginia, USA
}

\begin{abstract}
.
The astrometric capability of the Hubble Space Telescope Planetary Camera (WF/PC1) is investigated, motivated by a study of the internal velocity distribution of globular clusters. The astrometric accuracy of the HST PC will be determined ultimately by 1 ) the accuracy to which the aberrated images can be 'centered', and 2) the accuracy to which the distortions across the PC field can be modeled. A series of overlapping exposures of two clusters, NGC 6752 and M15, are utilized to examine these issues.

We have made use of maximum-likelihood image reconstruction to address the first issue, with good success. Reconstruction improves both the detectability and precision of the image centers. A preliminary exploration of the second issue, that of modeling the distortion across the PC field, is also presented, using positions derived from the multiple overlapping exposures.
\end{abstract}




\section{Introduction}

Among the Guaranteed Time Observing programs undertaken by the Space Telescope Astrometry Team, is a project designed to determine the distribution of internal velocity dispersion within several globular clusters. This is to be accomplished by measuring the relative proper motions of a sufficient number of stars over a range of mass and distance from the cluster core. This, in turn, will require the astrometric determination of precise relative positions of a large sample of stars at two separate epochs.

The primary astrometric instrument on board the Hubble Space Telescope is the Fine Guidance Sensor, whose superb astrometric performance is described elsewhere in this volume, (Benedict et al. 1994). A fundamental limitation of the FGS, however, is its 'serial' mode of operation which makes it unsuitable for measuring large numbers of stars. As an alternative, we have chosen to use the Planetary Camera for this task. A number of first-epoch exposures have been obtained using the WF/PC 1 in Planetary Camera mode. Second-epoch exposures are to be taken with the WF/PC 2. It has yet to be conclusively demonstrated that the required astrometric precision can be attained with the PC data. We describe here our present efforts at investigating the astrometric capabilities of the PC with specific attention given to our proposed study of the proper-motion distribution within globular clusters.

\section{Motivation}

Even with its aberrated images, the HST Planetary Camera allows imaging of individual main-sequence stars very near the cores of globular clusters. It is our goal to use the HST PC as an astrometric instrument to determine stellar proper motions within several globular clusters. The proper motions would yield:

1) internal velocity dispersions for these clusters,

2) virial mass estimates for these clusters,

3) kinematic distance estimates (statistical parallax), and

4) for a subgroup of the more nearby clusters; $i$ ) the radial and azimuthal velocity dispersions as a function of distance from the cluster center, and ii) the degree to which energy equipartition exists between various stellar mass groups within the clusters.

The clusters to be observed are 47 Tuc, M13, M22, NGC 6752, M15, M30, NGC 1851, and PAL 1. We have thus far obtained first-epoch exposures for the first five of these clusters. 


\subsection{OBSERVATIONS}

The Planetary Camera (WF/PC 1) consists of four $800 \times 800$ CCDs with a scale of $0.043 " /$ pixel. We adopt a nominal expected precision for relative stellar positions within individual frames of \pm 1 mas, i.e. 1/40 of a pixel. This would allow second-epoch exposures to be taken after five years. The resulting proper-motion errors would be \pm 0.3 mas $/ y r$ per 'plate pair', which corresponds to $7 \mathrm{~km} / \mathrm{s}$ for a cluster at $5 \mathrm{kpc}$ distance. Thus, the component due to measuring error of the observed velocity dispersion would be $3 \pm 0.3$ $\mathrm{km} / \mathrm{s}$, again assuming $\mathrm{d}=5 \mathrm{kpc}$. This value assumes five 'plate pairs' per star and 50 stars per velocity bin. Since the intrinsic velocity dispersions of globular clusters are typically 6 to $10 \mathrm{~km} / \mathrm{s}$, a measuring dispersion of $3 \pm 0.3$ $\mathrm{km} / \mathrm{s}$ is small enough and determined accurately enough to be confidently 'subtracted' from the total observed dispersion to yield the intrinsic cluster dispersion.

In order to calibrate the distortion across the PC field and to cover a large enough area to ensure a sufficient number of cluster star images, a strategy of using multiple-exposure patterns has been adopted. Two patterns are used, one a $5 \mathrm{x}$ pattern, the other, a $10 \mathrm{x}$ pattern. Within each pattern, the exposures are offset by 100 pixels in the $x, y$ system of the WF/PC. The cores of the program clusters are observed with 25-second and 100 -second exposures using the $10 \mathrm{x}$ and $5 \mathrm{x}$ patterns, respectively. The clusters are also observed with 1000-second exposures of the $5 \mathrm{x}$ pattern at positions corresponding to five and to ten times the cluster core radius. Observations are made in two passbands using the F555W and F785LP filters. The range in exposure times will hopefully allow us to measure stars roughly two magnitudes below the main-sequence turnoff for most of the clusters.

\section{Image Centering}

As stated earlier, the final astrometric precision of the relative positions derived from PC images will depend on the accuracy to which individual stellar images can be centroided and the degree to which the distortion across the PC field can be modeled. We have addressed the issue of optimum image centering with a variety of tests involving raw and reconstructed PC images, which we now describe in detail. Throughout the following discussion, the actual centroiding itself is done with a two-dimensional Gaussian fitting of the intensity profiles. 


\subsection{RAW IMAGES}

Previous tests, (Girard and van Altena, 1990), using synthetic PC frames and a limited number of early-observation frames of the R136 region showed that 2 mas relative positional accuracy could be achieved for well-exposed images using Gaussian fitting of the raw intensity profiles. The accuracy quickly fell off for fainter images and in crowded regions.

Although we are interested in an application involving crowded fields with low signal-to-noise images, (the globular cluster program), it is worth mentioning the centering precision we have recently achieved with a set of high signal- to-noise, isolated PC stellar images. This series of PC frames, taken in conjunction with FGS observations intended to monitor the longterm stability of the FGS instrument frame, was taken with the same pointing on consecutive orbits. The PC fields contain from two to three well-exposed stars. We have fit the 'raw' intensity profiles of the stars with our 2-D Gaussian centroider and estimated the centering precision from the deviations in the separation of star pairs. Based on 22 separation measures, the single- coordinate centroiding precision is 0.6 mas, ( 0.014 pixels)!

It must be stressed that this high level of repeatibility is thus far demonstrated only over consecutive HST orbits and with nearly identical pointing, (the individual star positions varied by $\approx 0.06$ to 0.20 pixels).

\subsection{RECONSTRUCTED IMAGES}

Image reconstruction of the aberrated PC images can make substantial improvements in qualitative appearance, and possibly recover faint star images from within the extended wings of nearby bright images. The question remains as to whether or not image reconstruction can retain or improve the astrometric information to be extracted from a PC image. We have performed a series of tests to help answer this question.

We have done so using the maximum likelihood image reconstruction code of Nunez and Llacer, (1990). Synthetic psf's generated by the Tiny Tim software package were used, and the frames were reduced assuming a uniform psf, (i.e., not a spatially varying psf across the PC field).

The first of these tests involved two non-program $\mathrm{PC}$ frames of the globular cluster NGC 6752, (a 40-second and 500-second exposure with F675W). Fifty-five (uncrowded) stars from PC-5 were selected by hand. The entire $800 \times 800$ pixel frame was restored using the maximum likelihood version of the Nunez and Llacer code. The reconstruction process is iterative, and was halted after 100 iterations. The restored intensity profiles were centered and the long exposure positions were transformed into those of the short exposure to determine the unit weight measuring error. The results are given in Table 1. 
TABLE 1. Long-to-short exposure reductions.

\begin{tabular}{lllll}
\hline & $\begin{array}{c}\sigma_{x} \\
\text { (mas) }\end{array}$ & $\begin{array}{l}\sigma_{y} \\
(\text { mas })\end{array}$ & $\begin{array}{l}\text { No. } \\
\text { Stars }\end{array}$ & $\begin{array}{l}\text { Mag. } \\
\text { Range. }\end{array}$ \\
\hline raw & 1.4 & 2.1 & 33 & 2.3 \\
psf @ $(200,600)$ & 1.4 & 1.2 & 39 & 2.9 \\
psf @ $(400,400)$ & 1.3 & 1.0 & 38 & 2.8 \\
psf @ $(600,200)$ & 0.8 & 0.8 & 42 & 3.3 \\
\hline
\end{tabular}

It should be noted that the 'raw' solution required the removal of many 'outliers' both bright and faint, thus the unit weight errors may be underestimated. The original target list consisted of 55 stars. Several of these were so saturated they did not center in any reductions. In addition, 8 to 10 very bright stars were eliminated due to a non-linear magnitude effect present.

These results suggest that 1 mas positional precision may be obtained with image reconstruction, for an uncrowded field of relatively well-exposed images.

\subsection{SUBSAMPLED RECONSTRUCTIONS}

Synthetic psf's may be generated on arbitrarily fine grids, allowing the reconstruction to be performed on a grid spacing smaller than that of the detector, one that better samples the psf. A subsampled reconstruction should be much less affected by the centering bias discussed above. We have used subsampled reconstructions, at subsampling factors of 1 through 5 , to explore its effect on the final centered positions.

Two additional PC exposures of NGC 6752 have been used for this part of our study, 100-second exposures in F555W and F785LP . A $128 \times 128$ subframe containing roughly 40 faint stellar images was extracted from PC 5. Tiny Tim psf's were calculated, in both filters, at the center of the subframe, and for each subsampling factor from 1 to 5 . The subframes were then processed through 80 iterations of the reconstruction code.

Twenty-five stars successfully centered on each of the $1 \mathrm{x}$ processed frames. The differences between the fractional pixel coordinates from the $1 \mathrm{x}$ and $5 \mathrm{x}$ reconstructions for these stars showed no indication of a systematic bias in the $1 \mathrm{x}$ fractional pixel coordinates, relative to the presumably accurate $5 \mathrm{x}$ coordinates, giving us somewhat more confidence in the error estimates quoted in Table 1.

In an attempt to determine just how the image coordinates "converge" 
from their $1 \mathrm{x}$ to their $5 \mathrm{x}$ values, linear transformations of the $\mathrm{Nx}$ positions were transformed into the $5 \mathrm{x}$ positions. Unexpectedly, the standard error does not decrease monotonically but is a minimum for the $3 \mathrm{x}$ transformation. If instead the transformations are performed into the $4 \mathrm{x}$ coordinates, the minimum appears for the $2 \mathrm{x}$ data! Clearly there is an artifact of the subsampling/reconstruction process rearing its ugly head here. This effect is currently being explored.

\section{Distortion}

The second of the two sources of astrometric uncertainty to be considered, that of distortion across the PC, has just begun to be explored. The positional displacements due to classical cubic distortion can be written

$$
\Delta \mathrm{x}=\mathrm{Dx}\left(\mathrm{x}^{2}+\mathrm{y}^{2}\right) \text {, and } \Delta \mathrm{y}=\mathrm{D} \mathrm{y}\left(\mathrm{x}^{2}+\mathrm{y}^{2}\right) .
$$

Comparing two exposures with a relative offset $(\delta \mathrm{x}, \delta \mathrm{y})$, the differential effect of the cubic distortion becomes quadratic in form

$$
\begin{aligned}
& \Delta \mathrm{x}_{2}-\Delta \mathrm{x}_{1}=3 \mathrm{D} \delta \mathrm{x}^{2}+2 \mathrm{D} \delta \mathrm{y} \mathrm{xy}+\mathrm{D} \delta \mathrm{x}^{2}, \\
& \Delta \mathrm{y}_{2}-\Delta \mathrm{y}_{1}=3 \mathrm{D} \delta \mathrm{y}^{2}+2 \mathrm{D} \delta \mathrm{x} \mathrm{xy}+\mathrm{D} \delta \mathrm{y} \mathrm{x}^{2} .
\end{aligned}
$$

Therefore, a general, quadratic, least-squares transformation between a pair of offset frames can yield the distortion coefficient. We have used five reconstructed, core exposures of M15 to test this method. Our preliminary results indicate that PC-5 of the WF/PC 1 exhibits classical cubic distortion of magnitude, $\mathrm{D}=-1.8 \pm 0.1 \times 10^{-5} \operatorname{arcsec}^{-2}$.

\section{References}

Benedict, G. F., McArthur, B., Nelan, E., Story, D., Whipple, A. L., Wang, Q., Jefferys, W. H., Duncombe, R. L., Hemenway, P. D., Shelus, P. J., van Altena, W. F., Franz, O. G., and Fredrick, L. W. (1994) 'HST, a generator of sub-millisecond of arc parallaxes' (This volume)

Girard, T. M. and van Altena, W. F., (1990) BAAS 22, 1277.

Nunez, J. M. and Llacer, J. (1990) Astrophys. and Space Sci. 171, 341. 